\title{
A Self-Adaptive Wireless Sensor Network Coverage Method for Intrusion Tolerance Based on Trust Value
}

\author{
Zuo Chen, ${ }^{1,2}$ Xue Li, ${ }^{1}$ Bing Yang, ${ }^{3}$ and Qian Zhang' \\ ${ }^{1}$ College of Computer Science and Electronic Engineering, Hunan University, Changsha, Hunan 410082, China \\ ${ }^{2}$ Institute of Information Engineering, Chinese Academy of Sciences, Beijing 100093, China \\ ${ }^{3}$ School of Education, Hubei University, Wuhan, Hubei 430062, China \\ Correspondence should be addressed to Bing Yang; yangbing@126.com
}

Received 18 August 2014; Accepted 8 October 2014

Academic Editor: Fei Yu

Copyright (C) 2015 Zuo Chen et al. This is an open access article distributed under the Creative Commons Attribution License, which permits unrestricted use, distribution, and reproduction in any medium, provided the original work is properly cited.

\begin{abstract}
The sensor is quite easily attacked or invaded during the process of the node coverage optimization. It is a great challenge to make sure that the wireless sensor network could still carry out a secure communication and reliable coverage under the condition of being attacked. Therefore, this paper proposes a network coverage method for intrusion tolerance based on trust value of nodes by combining the trust value model with the reliable coverage optimization. It first estimates trust value of nodes through which to regulate the perception radius and decision-making radius. Furthermore, this algorithm also combines the classical methods of wireless network coverage, such as GSO and PSO, to realize the networks coverage of invasive tolerant sensor. After comparing with the conventional single cover mechanism, it can improve the security and coverage rate of network under the condition of invasion. The simulation results verify the effectiveness of the algorithm.
\end{abstract}

\section{Introduction}

With the progress of science and technology in recent years, wireless sensor has a trend towards miniaturization, high efficiency, low power consumption, and so on and also can be applied to the field of radio communication to achieve costeffectiveness in mass production. Wireless sensor network node generally spreads to the region where people could not reach; it can organize itself as a network, search router, and detect the surrounding environment [1]. Due to the characteristics restriction of sensor node and the uncertain natural environment, the reliability and security of data is particularly important. Wireless sensor network, which can provide reliable communication and coverage under the condition of resource constraints and inevitable vulnerability along with the attacks and damage behavior, is urgently required.

In order to save energy, the researchers began to integrate coverage control function into the traditional sensor, so as to use fewer nodes as much as possible to complete the requirement of coverage. Regarding the problem of WSN node with vulnerable attacks and insecurity, the concept of trust was presented by the researchers to ensure the safety of the coverage area. At present, security routing technology based on trust management [2], safe fusion technology [3], and security time synchronization technology [4] have achieved some valuable research results, but up to now, the research field has little effective security cover mechanism based on trust management.

To improve the quality of coverage with the traditional covering algorithm and reduce effects of fault node on quality of coverage at the same time, researchers have worked out some reliable coverage algorithms. The literature [5] monitors the behavior of discard packets through some extra monitoring nodes in a network and helps failure nodes to send packets and support the sensor to transfer data to the sink node safely and accurately. The literature [6] proposed a reliable cover mechanism based on 2-Coverage, by increasing the redundancy cover to block out the single node failure, and therefore it can achieve the fault-tolerant effect. In order to avoid a single node failure which may cause the movement of whole mobile network nodes, literature [7] presented a scheduling scheme based on virtual coordinate. These studies 
of reliable cover mechanism mentioned above are mainly focused on the solution to reduce failure nodes on the quality of the cover. However, in many real situations, in addition to the node failure, network attack and invasion will also cause the decrease of the quality of the network coverage. For example, if the monitoring nodes in the network were captured by the enemy, turned into the malicious nodes, then the strengthening auxiliary mechanism of monitoring node will lose effectiveness. If part of the network was invaded, sensor data will gravely deviate from the real data [8], and these false data will probably lead to false alarms and consumption of limited network resources and cause serious consequence. So the researchers put forward the trust management mechanism, by monitoring the behavior of nodes to calculate the trust value of node, determine the behavior of the next term according to the trust. Trust management model is one of the effective methods to guarantee the network security in the case of wireless sensor network breach.

This paper proposes a coverage method for the invasive tolerant and adaptive sensor network based on trust value. Based on the coverage optimization in combination with the trust model, trust evaluation mechanism is able to identify malicious nodes and no cooperative nodes, resist network attack and invasion, improving coverage, and ensure the safety coverage of WSN.

The rest of this paper is organized as follows; the second part presents the system model; in the third part, trust management model is introduced and described; the fourth section presents the details of algorithm process; the fifth part is for simulation and analysis of simulation results; the last part is summary and outlook.

\section{System Model}

The coverage method for the invasive tolerant and adaptive sensor network based on trust value in this paper mainly includes two phases: the evaluation of node trust and the network coverage optimization. In the trust evaluation stage, the trust value of each node is calculated according to their own information and neighbor node information integration. In network coverage optimization stage, the node trust value is mapped to the radius changes and the traditional optimization of network coverage algorithm is applied to maximize reliable coverage.

2.1. Network Model. Suppose $N$ sensor nodes were randomly distributed in the $M * M$ square area and sensor node has key features as follows.

(1) All of the initial position and velocity of sensor nodes vary within a certain range of random initialization.

(2) Only one BS in the entire network node and BS node's energy is infinite.

(3) All sensor nodes in the network communication model and cognitive model are disc model; the original decision radius is twice the radius of perception, and all the radiuses are adjustable.
(4) Sensor nodes can be moved after deployment.

(5) Node is not equipped with GPS, but each node can know the current position information of itself.

(6) Each node establishes a trust table and a radius table for its neighbor nodes and records the change of trust value and the radius.

2.2. Network Coverage Model. $C^{\prime}$ is a subset of $C$, and $A\left(C^{\prime}\right)$ expresses the cover area of node set $C^{\prime}$, and regional coverage could be defined as $P_{\text {cov }}=A\left(C^{\prime}\right) / A(C)$. In practice, directly calculating the value of $A(C)$ is complex; to simplify the calculation, detection area $A$ will grid into $M * N$ points, the coordinates of the lattice are $(x, y)$, the distance from grid point to its sensor is defined as $d\left(c_{i}, p\right)=$ $\sqrt{\left(x-x_{i}\right)^{2}+\left(y-y_{i}\right)^{2}}$. Define the probability of grid points covered by sensor nodes as

$$
p\left(x, y, c_{i}\right)= \begin{cases}1 & \text { if } d\left(c_{i}, p\right) \leq r \\ 0 & \text { else. }\end{cases}
$$

When grid point $\left(x_{k}, y_{k}\right)$ covered by more than one sensor, mark it as covered state, and the probability of grid points been covered is defined as $z_{k}=\cup_{c^{\prime}} p\left(x_{k}, y_{k}, c_{i}\right)$, so the value of $z_{k}$ could only be 1 or $0 . A\left(C^{\prime}\right)=\sum_{M \times N} z_{k}$ and $A(C)=M \times N$, so area coverage is defined as

$$
P_{\text {cov }}=\frac{A\left(C^{\prime}\right)}{M \times N} .
$$

\section{Trust Management Model}

Comparing with the traditional security mechanism, trust management model has much more flexibility, scalability, and reliability. Meanwhile, it can complete the reliable authorization management, resource sharing, and security service via its establishment of a trust relationship among entities. Trust model is the main assessment according to "trust," by assessing behavior observation and interaction of individual record information, to get the evaluation of individual trust value computing model using appropriate mathematical calculation model. The trust model can be used to improve the security in open network environment based on the calculation and evaluation of node trust values for the implementation of the flexible adjustment of network security policy.

To this day, there are a lot of trust models for wireless sensor network (WSN) research. There are some secure routing technologies based on trust model. Wang et al. [9] point out a kind of wireless sensor network security routing algorithm without fixed infrastructure and with hardly detected malicious behavior which covers the safe trouble. The algorithm introduces the node credibility and also can establish secure routing and eliminates the malicious nodes of the network. Zhang et al. [10] put forward a credible wireless sensor network routing algorithm based on subjective logic. The algorithm takes full consideration of the node's credibility when established in route choice, 
to ensure the security of data transmission path constraint. Cheng et al. [11] put forward BT SR: an algorithm based on credible safety data fusion and routing. Respectively, from the perspective of the time and spatial correlation, it established a credible model based on similarity. This method solves the selective forwarding attacks and flood attacks in the process of information transmission to ensure the security of data transmission.

At present, there are few research results about reliable wireless sensor network covering algorithm. Shuhao and Xiaolon [12] and other researchers proposed an adaptive scheduling algorithm based on rotation of the trust nodes of the mesh. The algorithm is based on trust nodes scheduling which adapts rotation scheduling by the credibility of the nodes. Then find the virtual grid points that mesh formed, and quantify the trust value of each grid point. If the trust value cannot meet the requirements of safety cover, rescheduling to related nodes. After the calculation of trust value of the node for this algorithm, it should in turn calculate quantified credit of the trust grid points and thus become highly complicated. As the wireless sensor network typically consists of many small sensor nodes, and these sensor nodes only have limited communication bandwidth and energy, minimizing the complexity of the security covering algorithm can reduce energy consumption.

The trust model proposed in this paper takes different trust factors into consideration, including the observation from subjective one to the objective one and the recommendation of the third party. Neighbor nodes monitor each other, according to direct and indirect trust value from subjective one to the objective one, and then get comprehensive trust value. By adjusting the size of perception radius and decision radius according to comprehensive trust value to reduce the decision-making area and the coverage area of the low credibility nodes, in order to ensure the reliable coverage of the network.

3.1. Definition of Trust Factors. Assuming nodes $i$ and $j$ are neighbor nodes, node $i$ evaluates the trust of node $j$ from the aspects such as information communication, data integrity and consistency, and quantitative analysis of the various factors influencing the trust value.

3.1.1. The Consistency Factor $C F_{i, j}(t)$. To prevent malicious nodes forged packets, it is needed to analyze the space data consistency of the adjacent node. In wireless sensor networks, local neighbor nodes' data generally have high correlation. Subject $i$ monitored the packet content of object $j$, and comparing with its acquisition of data, if the difference of the two within a certain range, we can think that the assessment for monitoring objects has consistency between the subject and object. The consistency factor is as follows:

$$
C F_{i, j}(t)=\frac{C P_{i, j}(t)}{C P_{i, j}(t)+N C P_{i, j}(t)} .
$$

$C P_{i, j}(t)$ is the number of consistent data packets, and $N C P_{i, j}(t)$ is the number of inconsistent data packets.
3.1.2. Sending Rate Factor $S F_{i, j}(t)$. Subject $i$ evaluates and monitors the data sending situation of object $j$; if packet number is lower than the threshold limit TL, we can think it as a selfish node, and if the packet amount exceeds the maximum limit threshold $\mathrm{TH}$, we can think that it was in denial of service attack. Sending rate factor is as follows:

$$
S F_{i, j}(t)= \begin{cases}\frac{S P_{i, j}(t)-T_{L}}{E S_{i, j}(t)-T_{L}}, & S P_{i, j}(t)<E S_{i, j}(t) \\ \frac{T_{H}-S P_{i, j}(t)}{T_{H}-E S_{i, j}(t)}, & S P_{i, j}(t) \geq E S_{i, j}(t) .\end{cases}
$$

$S P_{i, j}(t)$ is the number of transmitting data packets within the period $t, E S_{i, j}(t)$ is the expected value of the total transmitted data packets within the period $t$, and it was preset by the base station according to the application. When sending rate factor is in the ideal range, node trust has a higher value.

3.1.3. Integrity Factor $I F_{i, j}(t)$. To prevent malicious nodes tampering and forwarding packets, we need to evaluate the integrity of the packets. After the source node sends data packets in a certain time, we monitor whether the next-hop node performed data forwarding correctly. Integrity factor is as follows:

$$
I F_{i, j}(t)=\frac{I P_{i, j}(t)}{D T P_{i, j}(t)} .
$$

$I P_{i, j}(t)$ is the complete forward package number and $\operatorname{DTP}_{i, j}(t)$ is the number of packets that subject $i$ needs object $j$ to forward.

3.1.4. Radius Factor $R A(t)$. The trust between nodes boils down to whether or not to transmit data packets. In order to prevent malicious nodes transmit more non-normal data, right amount to reduce the size of perception radius and decision radius of low trust value nodes. Radius factor is as follows:

$$
R A(t)=\frac{R(t)}{R_{0}} .
$$

$R(t)$ is the radius of node in the period $t$ and $R_{0}$ is original radius.

3.1.5. The Time Factor $T F(t)$. Due to that fact that node trust value is combination of trust record and current observation information, the time factor could be joined to analyze the correlation with the context of the trust value, to reflect trust value with time attenuation effect. If the time factor is too large, the trust value is affected by the history too much and the evaluation of the node might be wrong, while if the time factor is too small, the trust value may have excessive dependence on a single time period. Therefore, we need to make different time factor according to different security levels.

3.2. The Calculation of Trust Value. According to the specific application requirements, evaluating node $i$ monitoring part of or all of the trust factors, evaluate object $j$ 's direct trust 
values of $D T_{i, j}(T)$ by method of weighted average. In all of the defined trust factors, $S F_{i, j}(T)$ and $C F_{i, j}(T)$ mainly consider the rationality of the $I F_{i, j}(t)$ amount of data packet transmission and related content. They were involved in evaluation object data forwarding, part of forwarding the data packet integrity, and relative surplus energy. Suppose that evaluation of the trust value monitoring all trust factors mentioned above, and the historical trust of the previous cycle expressed as $D T_{i, j}(T-1)$ and the corresponding radius is $R A(T-1)$, calculation formula of direct trust value can be defined as

$$
\begin{aligned}
D T_{i, j} & (t) \\
= & T F(t) \\
& \times\left(\omega 1 S F_{i, j}(t) C F_{i, j}(t)+\omega 2 I F_{i, j}(t)+\omega 3 R A(t-1)\right) \\
& +(1-T F(t)) D T_{i, j}(t-1) .
\end{aligned}
$$

$\omega_{1}, \omega_{2}, \omega_{3}$ are the weighted coefficients, which could be adjusted according to specific circumstances, and

$$
\omega_{1}+\omega_{2}+\omega_{3}=1 \text {. }
$$

The interaction between the subject of $i$ and the object of $j$ is not only direct but also indirect through the common neighbor. So the node $i$ trust calculation includes direct trust and indirect trust value. Indirect trust is limited to $i$ and $j$ common neighbor node trust transfer. The indirect trust value is as follows:

$$
I T_{i, j}(t)=\frac{\sum_{k=1}^{s} D T_{i, k}(t) D T_{k, j}(t)}{s} .
$$

$k$ is one of $i$ and $j$ 's common neighbor nodes and $s$ is the total number of the common neighbor nodes.

So the evaluation of $i$ comprehensive trust degree on object $j A T_{i, j}(t)$ is as follows:

$$
A T_{i, j}(t)=\lambda D T_{i, j}(t)+(1-\lambda) I T_{i, j}(t) .
$$

$\lambda$ is a comprehensive trust in direct trust degree of dependence and it can be adjusted for specific application.

3.3. Node Radius Adjustment of the Trust. With the assessment of trust between nodes, we can accurately judge the malicious nodes in the network. In wireless sensor networks, in order to mitigate what the malicious node brings, the sensing radius and the radius of decision nodes should be regulated according to the trust degree of node, in order to make the malicious nodes communication and the coverage reduction in the network. Each node can establish a radius table to its neighbor node, to record their radius change. When a node $j$ was judged to be malicious nodes, the neighbor node will update its radius as follows:

$$
R^{\prime}=R_{0} f\left(\frac{\sum_{i=1}^{n} A T_{i, j}(t)}{n}\right) .
$$

$R_{0}$ is the original radius, including sensing radius and radius of coverage. Node $i$ is the neighbor of node $j ; n$ is the total number of neighbors. Function $f$ can be changed according to the need of different network while we used the linear function in this paper. After updating the radius, the radius of neighbor nodes list will also be updated.

\section{Method Description}

Swarm intelligence algorithm [13] is inspired by the nature of biological behavior, such as particle swarm optimization, ant colony optimization, and firefly algorithm. They have their own unique strengths but also have their own defects. In order to overcome the defect of the original algorithm, combining two or more algorithms together has become the trend of the research.

4.1. The Basic PSO and GSO Algorithm. In 1995, American psychologist Kennedy and electrical engineer Eberhart introduced an algorithm of particle swarm optimization (PSO) [14]. It is inspired by the migration and clustering of the birds during their foraging. In the original algorithm, its argument is consistent, and there is limitation in the solving process. Considering the basic particle swarm optimization, Jianping et al. [15] introduced inertia weight $\omega$, which decreases linearly in the original formula, and formulated basic PSO algorithm. The algorithm [16] supports that every individual is a particle without volume and quality, flying at a certain speed in the search space, and adjusts its velocity dynamically according to the comprehensive analysis of the flying experience of individual and group.

In 2005, Luo et al. promoted a new swarm intelligence heuristic computing technology: GSO (glowworm swarm optimization) [17]. GSO algorithm developed a multipoint parallel global random search strategy based on the behavior of group [18]. High speed and efficiency of capturing the extreme point make GSO have strong versatility [19]. The significant factor of finding the optimization in firefly algorithm is the brightness and attraction. Brightness depends on the current position and the objective function value, which is higher when the position is better. At the same time, the attraction is influenced by the brightness, which means the brighter fireflies have the stronger attraction and also can attract those less bright fireflies. With the increase of distance, the brightness and attraction of media decrease after absorbing fluorescence. In all, there are four stages in the GSO process [20]: fluorescein update, firefly movement, firefly position update, and firefly neighbor radius update.

4.2. Improved Algorithm PGSO. PGSO adopts the method of series which combines particle swarm optimization (PSO) algorithm and the firefly algorithm. Firefly algorithm is able to discover global optimal solutions and local optimal solutions of the search space; the disadvantage is the high time cost and accuracy is not high. Particle swarm optimization has the advantage of fast convergence speed and high calculation accuracy; its shortcoming is ease of falling into local optimum. After merger of two algorithms, precision 
of solution is higher than particle swarm optimization and firefly algorithm and helps to overcome the problem of falling into local optimum.

Assuming the improved algorithm PGSO's particle swarm is made up of $m$ particle. Particle target search space is composed of $n$ fireflies. $x_{i}=\left(x_{i 1}, x_{i 2}, \ldots, x_{i n}\right)$ is the position vector of the $i$ th particle $(i=1,2, \ldots, m) ; v_{i}=\left(v_{i 1}, v_{i 2}, \ldots, v_{i n}\right)$ is the $i$ th particle velocity, on behalf of the next iteration particle moving distance; $p_{i}=\left(p_{i 1}, p_{i 2}, \ldots, p_{i n}\right)$ is the optimal value in the search history of the $i$ th particle, namely, the local optimal value; $p_{g}=\left(p_{g 1}, p_{g 2}, \ldots, p_{g n}\right)$ is the optimal value in the search history of the whole particle swarm, namely, the global optimal value. Each iteration process is as follows.

(1) In the iterative optimization process, firstly, update the particle velocity and position based on particle swarm optimization (PSO) algorithm as follows:

$$
\begin{gathered}
v_{i d}^{(k+1)}=\omega v_{i d}^{k}+c_{1} r_{1}\left(p_{i d}-x_{i d}^{k}\right)+c_{2} r_{2}\left(p_{g d}-x_{i d}^{k}\right) \\
x_{i d}^{(k+1)}=x_{i d}^{k}+v_{i d}^{(k+1)} .
\end{gathered}
$$

$i=1,2, \ldots, m ; d=1,2, \ldots, n ; d$ is the current dimension for target search space; $k$ is the current number of iterations; $r 1$ and $r 2$ are random numbers in $[0,1]$ which obey uniform distribution; $c 1$ and $c 2$ are artificial learning factors. Inertia weight $\omega$ is as follows:

$$
\omega=\omega_{\max }-\frac{\omega_{\max }-\omega_{\min }}{\text { iter }_{\max }} \times k
$$

$\omega_{\max }$ is the initial weights; $\omega_{\min }$ is the weight of termination; iter $_{\max }$ is maximum number of iterations, and $k$ is the current number of iterations.

(2) Applying the firefly algorithm iterative to update searching space. The firefly luciferin is updated as follows:

$$
l_{i}(t+1)=(1-\rho) l_{i}(t)+\gamma J\left(x_{i}(t+1)\right) .
$$

$l_{i}(t)$ is the fluorescein concentration of firefly $i$ with $t$ iteration; $\rho(O<\rho<1)$ is the fluorescein concentration attenuation coefficient; $J\left(x_{i}(t)\right)$ is the objective function values of node $i$ with $t$ iteration. The objective function is based on the coordinates of node $i$. Take the objective function as

$$
J\left(x_{i}(t+1)\right)=\sum_{j=1}^{k} \frac{l_{i}(t+1)}{d_{i j}(t+1)} .
$$

In the formula

$$
d_{i j}=\sqrt{\left(x_{i}-x_{j}\right)^{2}+\left(y_{i}-y_{j}\right)^{2}}
$$

$$
\begin{aligned}
j \in & N_{i}(t+1) \\
& =\left\{j: d_{i j}(t+1)<r_{d}^{i}(t+1),\left\|x_{j}(t)-x_{i}(t)\right\|>0\right\} .
\end{aligned}
$$

$d_{i j}$ is the Euclidean distance between nodes $i$ and $j$ and $j$ is the adjacent nodes of node $i$ which is in the perception of radius and different to $i . r_{d}^{i}(t)$ is the radius of the adjacent decision domain of node $i$ with $t$ iteration. According to the formula (13) we update the neighbors fireflies' storage of $r_{d}^{i}(t)$, narrowing the scope of low trust fireflies decision making.

(3) Computing the probability of firefly move to their neighbor whose fluorescein concentration is lower than itself we have

$$
P_{i j}=\frac{l_{i}(t)-l_{k}(t)}{\sum_{k \in N_{i}(t)}\left(l_{i}(t)-l_{k}(t)\right)} .
$$

In the formula

$$
k \in N_{i}(t)=\left\{k: d_{i k}(t)<r_{d}^{i}(t), l_{i}(t)<l_{k}(t)\right\} .
$$

$K$ is the adjacent nodes of node $i$ whose fluorescein concentration is lower than $i$ and in the perceived radius of $i$ with $t$ iteration. $d_{i k}(t)$ is the Euclidean distance between $i$ and $k$ with $t$ iteration.

(4) Location updates after the firefly moved is

$$
x_{i}(t+1)=x_{i}(t)+s *\left(\frac{x_{j}(t)-x_{i}(t)}{\left\|x_{j}(t)-x_{i}(t)\right\|}\right) .
$$

$x_{i}(t)$ is the space position of node $i$ in $n$ dimension and $s$ is step length of location update iteration. $\| x_{j}(t)-$ $x_{i}(t) \|$ is Euclidean distance.

(5) Updating each particle, nest the firefly algorithm in particle swarm optimization algorithm to keep accurate particle. After updating $m$ particles, update individual optimal values and global optimal value according to the coverage.

4.3. The Intrusion Tolerance Security Coverage Method Based on PGSO Algorithm. When the network is under attack, or part of the node is invaded, sensor data gravely deviates from the real data and even breaks the authenticity of the data and may also infect neighbor nodes, consume limited network resources, and cause a serious consequence. The intrusion tolerance security coverage method is based on PGSO algorithm by using the above mentioned trust management model and combining PGSO iterative process to achieve the effect of adaptive adjustment cover as follows.

(a) After initialization of $m$ particles, according to formula (10) in trust models, calculate each node's trust, again by formula (11) to adjust the radius list of the neighbor node. Reduce the low trust nodes' coverage areas.

(b) According to formula (11) to update the $r_{d}^{i}(t)$ stored in the neighbor nodes' list, narrow the scope of low trust fireflies decision making.

(c) According to formula (10) compute node's trust after the update particle round. Regulate the nodes' radius and coverage according to formula (11) and then start a new iteration 3 . 


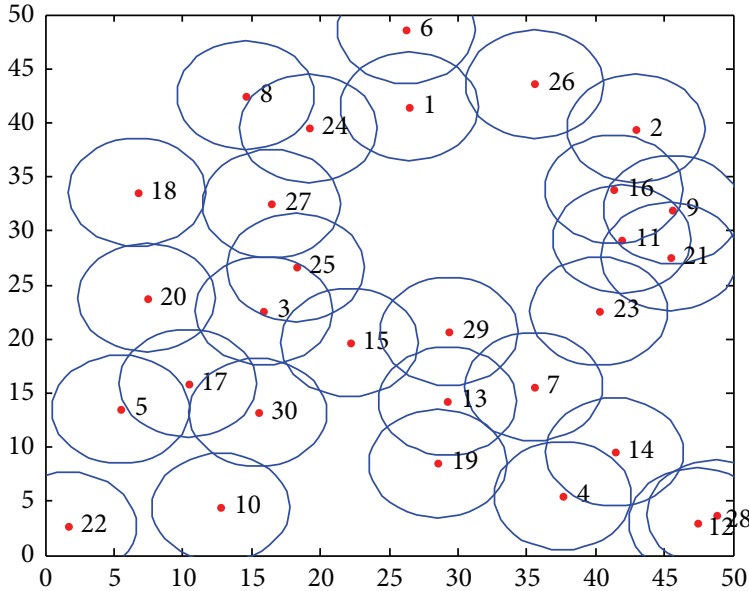

(a) Initial random distribution of sensor nodes

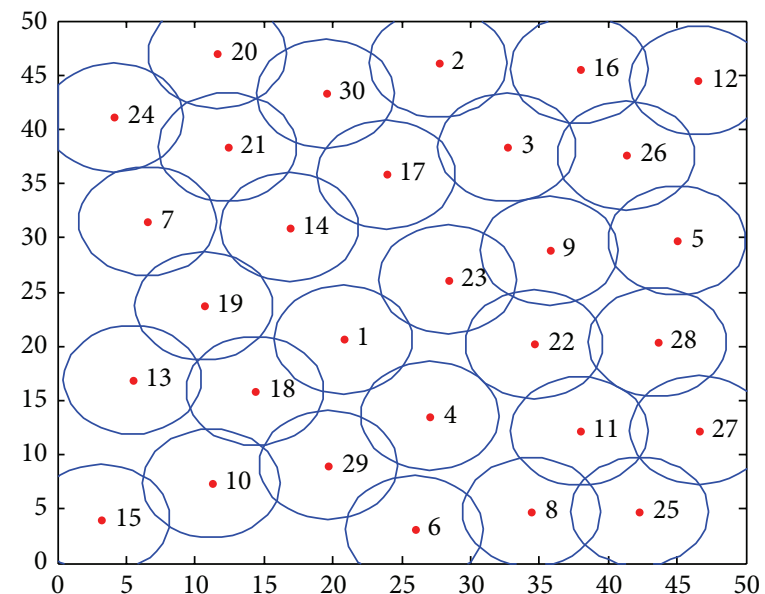

(b) PGSO node deployment optimization

FIGURE 1: PGSO to 30 nodes deployment optimization algorithm.

\section{Simulation}

5.1. PGSO Algorithm Simulation and Performance Analysis. In order to verify the validity of the PGSO algorithm, we evaluate the algorithm in MATLAB environment, so that we may improve the algorithm itself through the related simulation test and comparison. Set the particle size of particle swarm optimization (PSO) algorithm as $m=40$, the number of nodes in each particle as $n=30$; that is, the number of the fireflies is 30 , the largest number of iterations is iter $r_{\max }=200$, the initial value of perceived radius is $5 \mathrm{~m}$, the initial value of radius of decision is $10 \mathrm{~m}$, and learning factor is $c_{1}=c_{2}=2$. Linear decreasing inertia weight $\omega$, the initial weights $\omega_{\max }=0.9$, weight of the termination $\omega_{\min }=0.4$, the attenuation coefficient of fluorescein in GSO algorithm $\rho=0.9$, fitness extraction ratio $\gamma=0.1$. In the $50 \mathrm{~m} *$ $50 \mathrm{~m}$ square monitoring area, mesh point size is set as $0.5 \mathrm{~m} *$ $0.5 \mathrm{~m}$. Distribute 30 mobile sensor nodes in the monitoring area randomly and use PGSO algorithm to do the simulation. (a) and (b) in Figure 1 present node distribution simulation diagram before and after optimization, respectively.

As can be seen from the above, the network coverage strategy put forward in this paper can give more reasonable node deployment optimization scheme, improving the network coverage at the same time. From the simulation results, we can see node deployment from the complicated state optimal operation to the uniform distribution, and overlapped covered area by each other is relatively small. In order to further verify the validity of the algorithm, under the experimental environment, we, respectively, do the experimental simulation for PSO algorithm, GSO algorithm, and PGSO optimization algorithm. The simulation results are as shown in Figure 2.

As can be seen from Figure 2, network coverage of PGSO optimization algorithm in each iteration step is always greater than both the GSO algorithm and PSO algorithm. Furthermore, in order to test whether PGSO can give better network coverage optimization, under the different condition

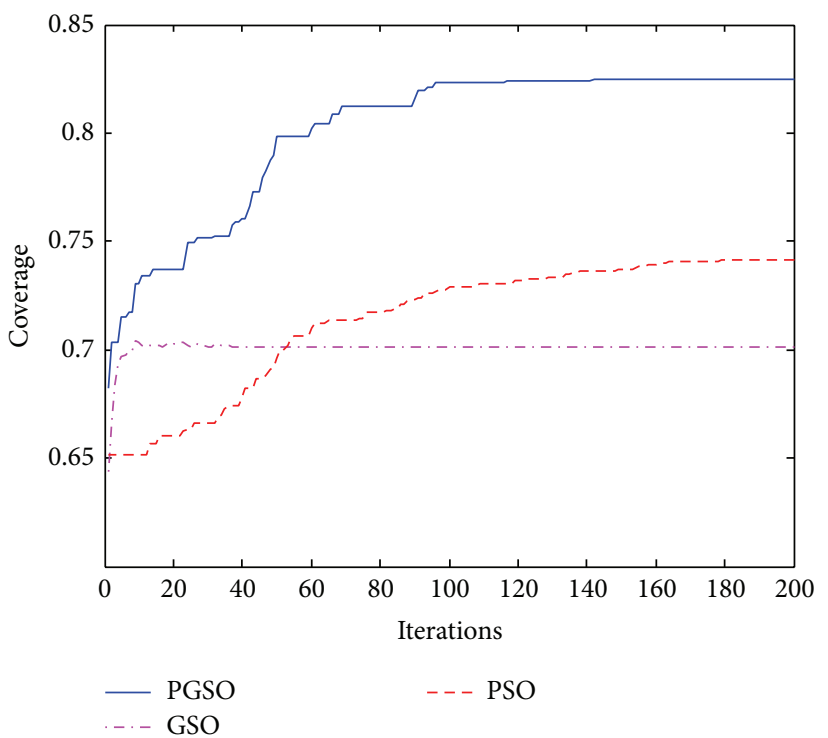

FIGURE 2: Network coverage comparison chart.

of the network node number, we set node numbers 10, 15, $20,25,30,35,40$, and 45, separately, and adopt the GSO algorithm, PSO algorithm, and PGSO algorithm to make simulations. These results of each algorithm under different node number were taken using average network coverage of 10 times of simulation. The simulation results are shown in Figure 3. It can be seen from Figure 3, when the network node is at the same time, coverage of PGSO algorithm is always greater than that of GSO and PSO algorithms, and advantage will be more apparent especially in the larger network.

5.2. PGSO Algorithm Based on Trust Instance Simulation and Performance Analysis. This paper joins the trust model and PGSO to realize reliable coverage. In order to verify its 


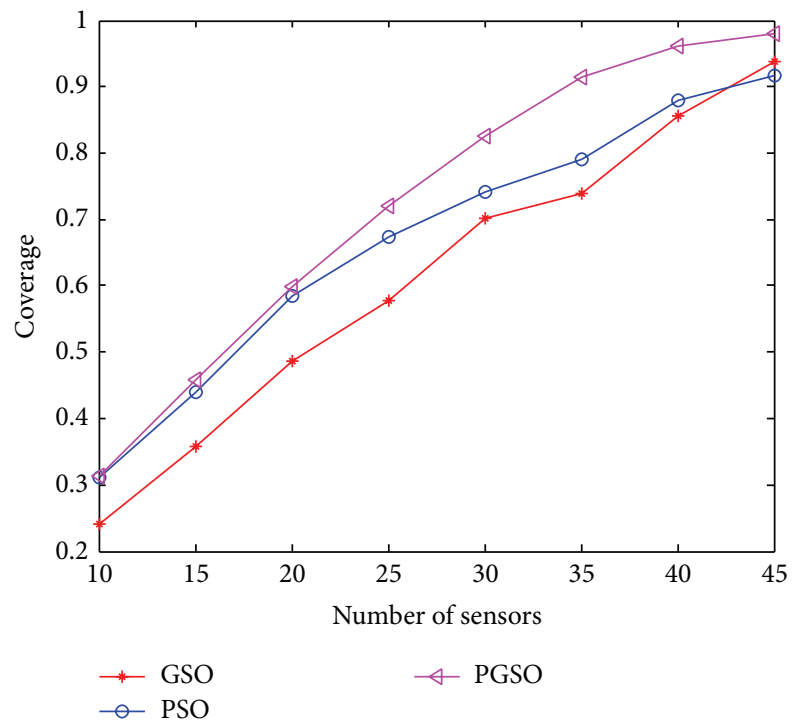

FIGURE 3: Coverage changes along with the network size.

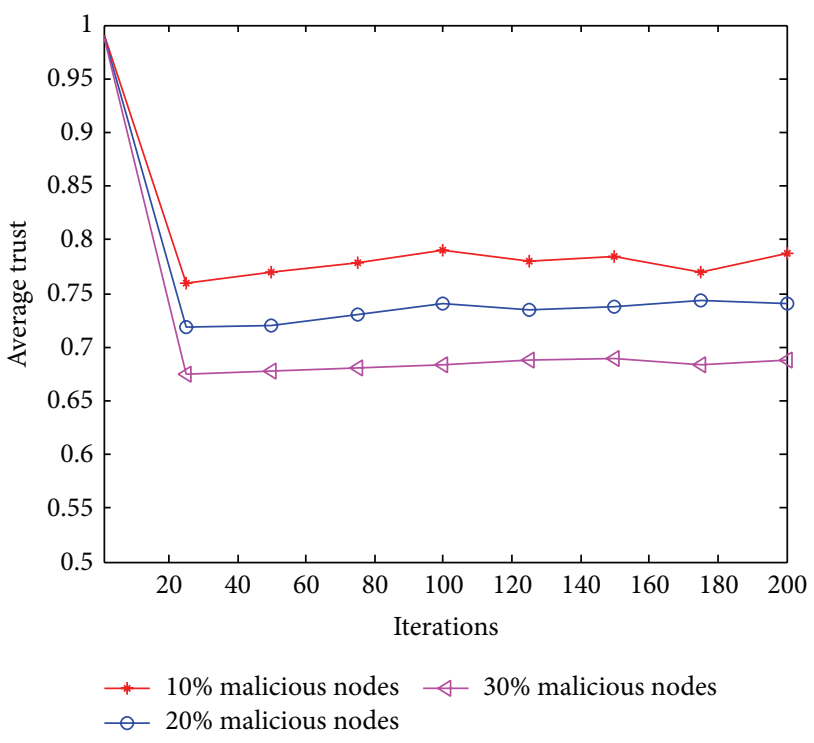

FIGURE 4: Average trust value changes of network node.

effectiveness, we take evaluated node trust value into account including trust factor such as data consistency, sending rate, integrity, and time. Set the particle size as $m=40$; each particle has a number of nodes $n=30$. A different number of nodes turn into malicious nodes by artificial random setting, and malicious nodes will send false data information or tamper with the forwarding packets. Monitor the change of trust value and the radius of normal nodes, malicious nodes, and network coverage in the meantime. By setting the malicious nodes proportion as $10 \%, 20 \%$, and $30 \%$, respectively, in the simulation experiment, node average trust values are shown in Figure 4. All nodes trust values are set to 1 at the beginning of the figure; that is, all the nodes are considered credible. By detecting average trust value of nodes
TABLE 1: Comparison of coverage in different network under varying invasion degree.

\begin{tabular}{lccc}
\hline \multirow{2}{*}{ Algorithm } & \multicolumn{3}{c}{ Invasion ratio } \\
& $10 \%$ & $20 \%$ & $30 \%$ \\
\hline PGSO1 (30) & 0.7595 & 0.7101 & 0.6411 \\
PGSO2 (30) & 0.7718 & 0.7232 & 0.6559 \\
PGSO1 (40) & 0.8611 & 0.8283 & 0.7617 \\
PGSO2 (40) & 0.8977 & 0.8635 & 0.8112 \\
PGSO1 (50) & 0.9299 & 0.8855 & 0.8314 \\
PGSO2 (50) & 0.9474 & 0.9037 & 0.8525 \\
\hline
\end{tabular}

in the network every 25 times of the iterative process, the average trust value of nodes tends to be stable during the subsequent iteration process. The average node trust value is lower when there are more intrusion nodes in the network.

Under the condition of the experimental environment mentioned above, a simulation of a set of comparative experiments is necessary. Contrast test does not include the node trust mechanism model, because in many cases, in order to guarantee the security of the network, once it detects malicious nodes, the malicious nodes will be placed as a dormant state or isolated state. For the sake of clearness, PGSO1 is short for the malicious node dormancy or segregated algorithm and PGSO2 is short for PGSO algorithm based on trust model. Set the proportion of malicious node as $10 \%, 20 \%$, and $30 \%$, respectively, in the simulation experiment. The simulation results are as shown in Figures 5 and 6.

In Figure 5, black triangle mark is invaded node, and the red dot is normal node. From a series of contrast figures, it can be found obviously that the coverage method for the invasive tolerant and adaptive sensor network based PGSO is much better. It has a larger coverage and also can ensure the security of network.

Seen from Figure 6, network coverage of PGSO2 algorithm in each iteration step is greater than that of PGSO1 basically. And in the case of node invasion increase, the drop of coverage is much smaller than that of isolated model. To further show that the coverage method for the invasive tolerant and adaptive sensor network based on trust value can give a more reasonable network coverage optimization scheme, consider the condition of the network node number is 30, 40, and 50. Respectively use PGSO1 and PGSO2 to simulate 10 times, and take the average network coverage into comparison. The simulation results are shown in Table 1. PGSO1 (30) in Table 1 expresses the PGSO1 algorithm simulation result when the node number of network is 30 . And by this analogy, it can be seen from the data in the table that with the increase of network scale, PGSO2 has better performance than PGSO1. Therefore, PGSO2 has a tendency to do better in a large network.

\section{Conclusion and Future Works}

In order to guarantee the security of network under the maximum effective coverage, this paper proposes a network coverage method for the invasive tolerant sensor based 
PGSO1 optimization distribution

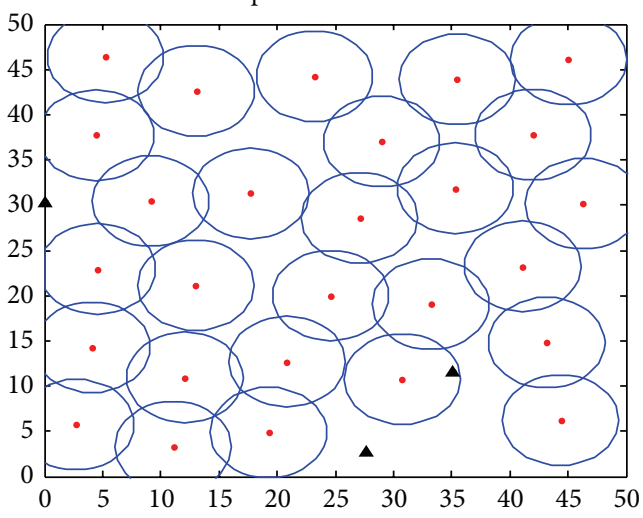

PGSO2 optimization distribution

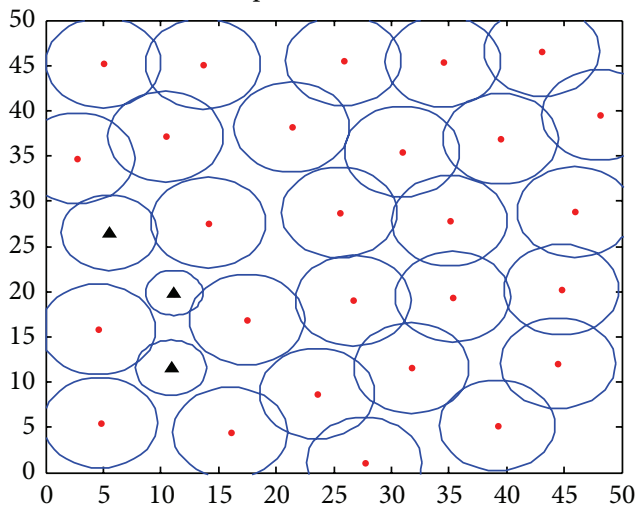

(a) $10 \%$ of nodes suffer the invasion
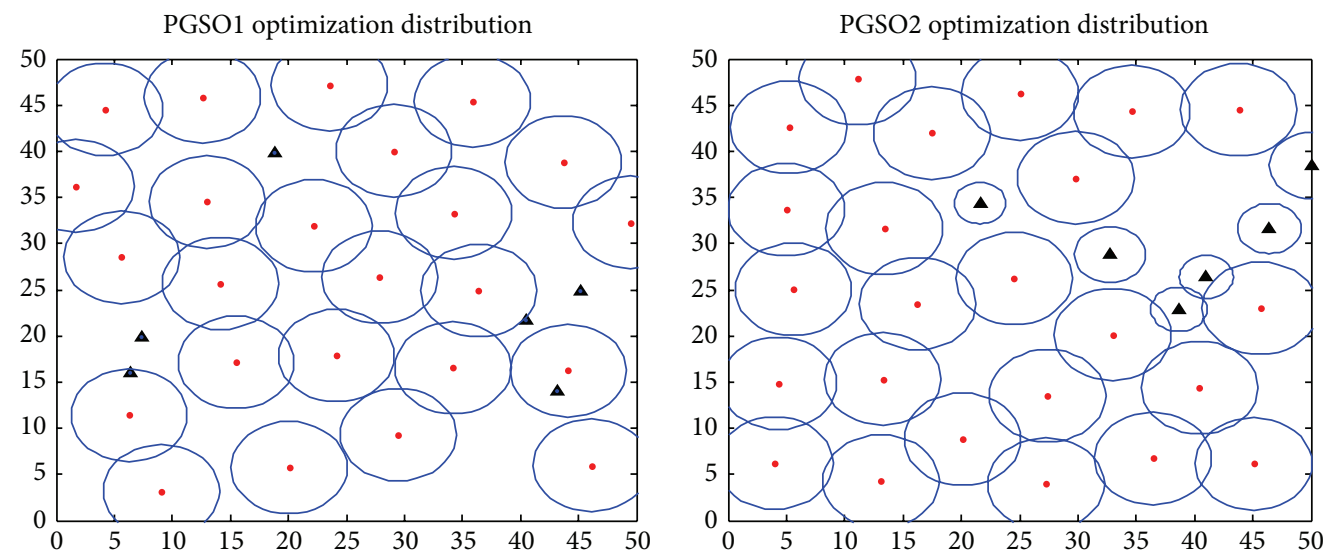

(b) $20 \%$ of nodes suffer the invasion
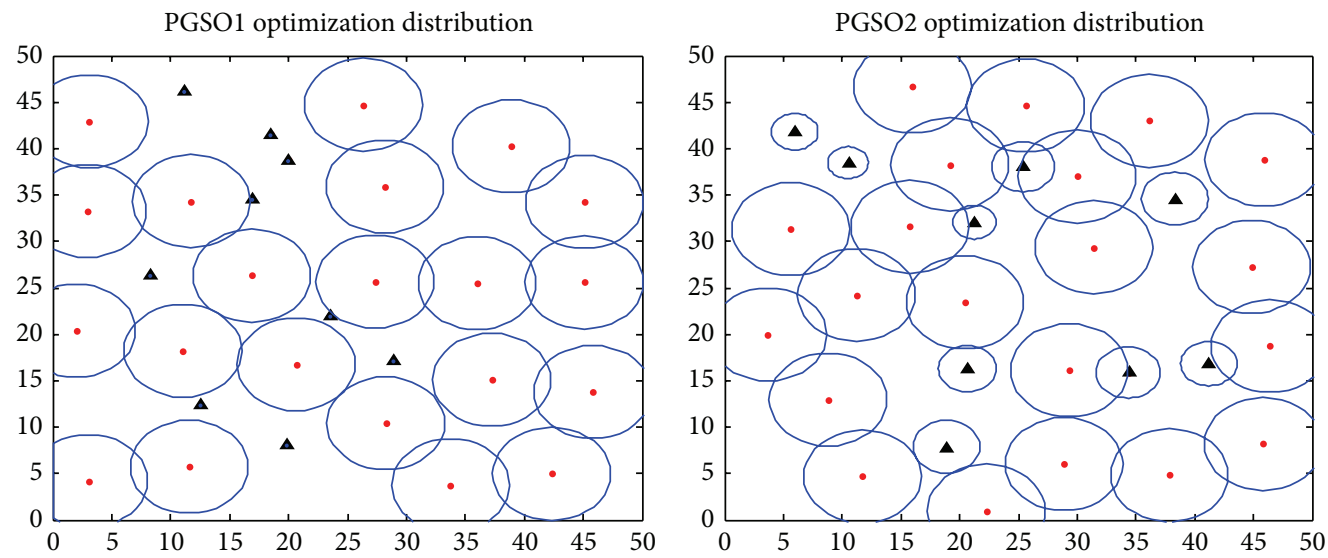

(c) $30 \%$ of nodes suffer the invasion

Figure 5: Contrast of algorithm optimization.

on trust value of nodes. Based on the consistency, data transmission rate, integrity, and time factor, nodes could estimate each other's trust value and next adjust radius of node according to the different trust value and, at the same time, integrate this trust model into the optimization algorithm of traditional coverage method, PGSO, making sure the network can adjust to the maximum reliable coverage when under invasion. The simulation results show that the coverage rates of PGSO algorithm and GSO algorithm with PSO both have increased evidently. And based on the same reliable coverage rate, compared with the traditional method of isolating malicious nodes, the PGSO algorithm integrated with trust value performs better, especially under a large scale of nodes. 


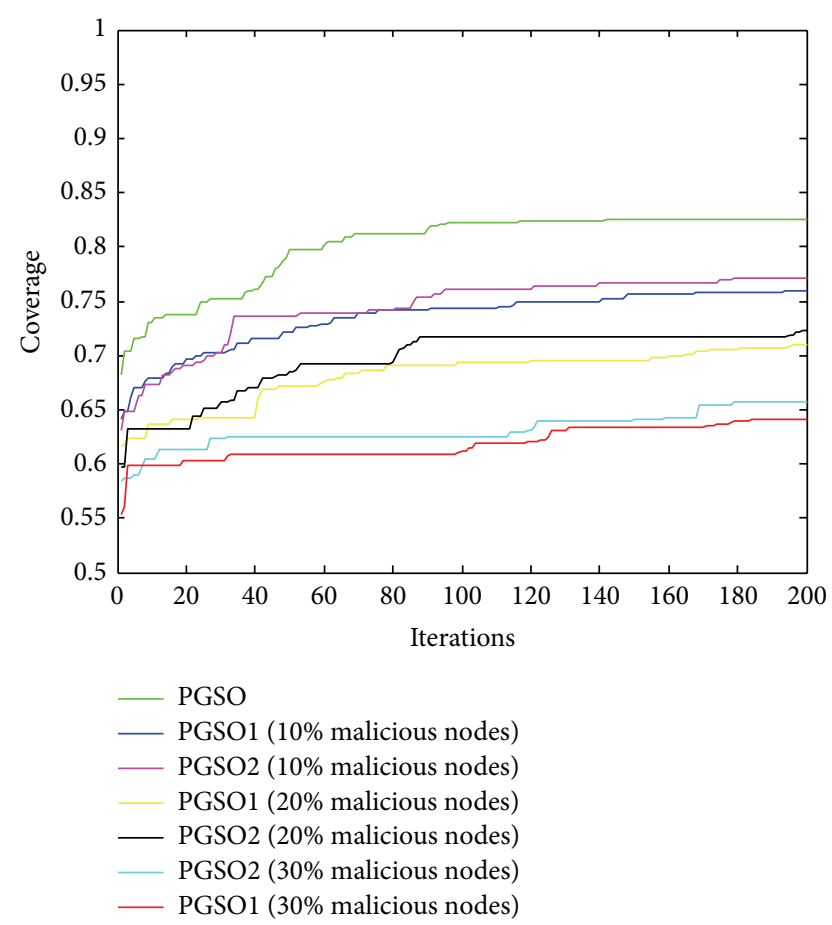

Figure 6: The process of convergence when the node suffers invasion.

The current problems focused on the following three aspects. First of all, there are many types of node attacks, for example, replay attacks and so on, so the trust factor in the trust value model needs to be improved. Secondly, when the node is judged to be a malicious one, more experiments and follow-up studies are needed in order to find a formula with more appropriate trust value and radius corresponding regulation. In addition, due to the energy limitation of mobile sensor network node, it is of great significance to extend the life coverage of the reliable network appropriately when considering the node trust values and energy consumption.

\section{Conflict of Interests}

The authors declare that there is no conflict of interests regarding the publication of this paper.

\section{Acknowledgments}

This work was sponsored by the Natural Science Foundation of Hunan Province, China (13JJ3091, 14JJ3062), National Nature Science Foundation, China (61202462, 61300036), and the Fundamental Research Funds for the Central Universities, China.

\section{References}

[1] I. F. Akyildiz, W. Su, Y. Sankarasubramaniam et al., "Wireless sensor networks: a survey," Computer Networks, vol. 38, no. 4, pp. 393-422, 2002.
[2] K. Nagarathna, Y. B. Kiran, J. D. Mallapur, and S. Hiremath, "Trust based secured routing in wireless multimedia sensor networks," in Proceedings of the 4th International Conference on Computational Intelligence, Communication Systems and Networks (CICSyN '12), pp. 53-58, IEEE Press, Piscataway, NJ, USA, July 2012.

[3] Y. Chen and J. Shu, "Wireless sensor networks security issues as smart materials systems," Applied Mechanics and Materials, vol. 63-64, pp. 497-501, 2011.

[4] Z. Y. Tao and M. Hu, "Time synchronization algorithm based on hierarchical structure in wireless sensor network," Journal of Computer Applications, vol. 32, no. 6, pp. 1513-1515, 2012.

[5] H. Gobjuka and Y. Breitbart, "Discovering network topology of large multi subnet ethernet networks," in Proceedings of the 32nd IEEE Conference on Local Computer Networks (LCN '07), pp. 428-435, October 2007.

[6] L. Qionglin, "Inclusion-exclusion principle and application," China Science and Technology Information, vol. 20, no. 8, pp. 5859, 2012.

[7] A. Francy Golda, S. Aridha, and D. Elakkiya, "Algorithmic agent for effective mobile robot navigation in an unknown environment," in Proceedings of the International Conference on Intelligent Agent and Multi-Agent Systems (IAMA '09), pp. 1-4, Chennai, India, July 2009.

[8] W. R. Pires, T. H. P. Figueiredo, H. C. Wong, and A. A. F. Loureiro, "Malicious node detection in wireless sensor networks," in Proceedings of the 18th International Parallel and Distributed Processing Symposium, 2004.

[9] C. Wang, X. Y. Jia, and Q. Lin, "Trust based secure routing algorithm for wireless sensor networks," Journal on Communications, vol. 29, no. 11, pp. 105-112, 2008.

[10] L. Zhang, L. Li, and C. Li, "A wireless sensor network routing algorithm based on subjective logic trusted," Wuhan University of Technology, vol. 33, no. 1, pp. 75-78, 2009.

[11] Z. Cheng, Z. Ming-Zheng, and X. Jinsheng, "BTSR: A behaviorbased safety data fusion and routing algorithm credible," Journal of Computer Applications, vol. 28, no. 11, pp. 2820-2823, 2008 .

[12] D. Shuhao and L. Xiaolon, "Reliable coverage algorithm in wireless sensor networks based on grid trust," Application Research of Computers, vol. 31, no. 1, pp. 253-256, 2014.

[13] E. Bonabeau, M. Dorigo, and G. Theraulaz, Swarm Intelligence: From Natural to Artificial Systems, Oxford University Press, Oxford, UK, 1999.

[14] J. Kennedy and R. Eberhart, "Particle swarm optimization," in Proceedings of the IEEE International Conference on Neural Networks, pp. 1942-1948, December 1995.

[15] L. Jianping, X. Li, and C. Minrong, "SFLA the Markov model and its convergence analysis," Acta Electronica Sinica, vol. 38, no. 12, pp. 2875-2880, 2010.

[16] E. Sun, "A survey on clustering routing protocols based on PSO in WSN," TELKOMNIKA Indonesian Journal of Electrical Engineering, vol. 12, no. 7, 2014.

[17] J.-P. Luo, X. Li, and M.-R. Chen, “The Markov model of shuffled frog leaping algorithm and its convergence analysis," Acta Electronica Sinica, vol. 38, no. 12, pp. 2875-2880, 2010.

[18] K. N. Krishnanand and D. Ghose, "Theoretical foundations for rendezvous of glowworm-inspired agent swarms at multiple locations," Robotics and Autonomous Systems, vol. 56, no. 7, pp. 549-569, 2008. 
[19] K. N. Krishnanand and D. Ghose, "Glowworm swarm optimization:a new method for optimizing multi-modal functions," International Journal of Computational Intelligence Studies, vol. 1, no. 1, pp. 93-119, 2009.

[20] K. Huang and Y. Zhou, "Improved variation step adaptive GSO algorithm," Computer Engineering, vol. 38, no. 4, pp. 185-187, 2012. 

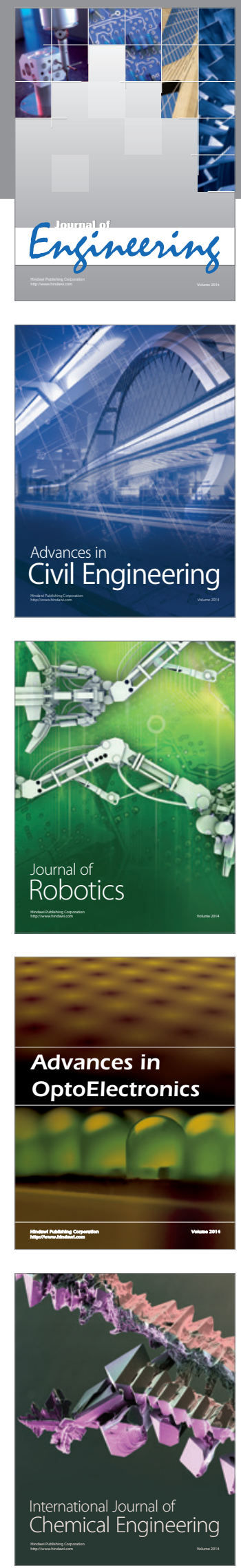

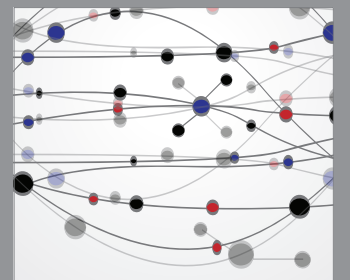

The Scientific World Journal
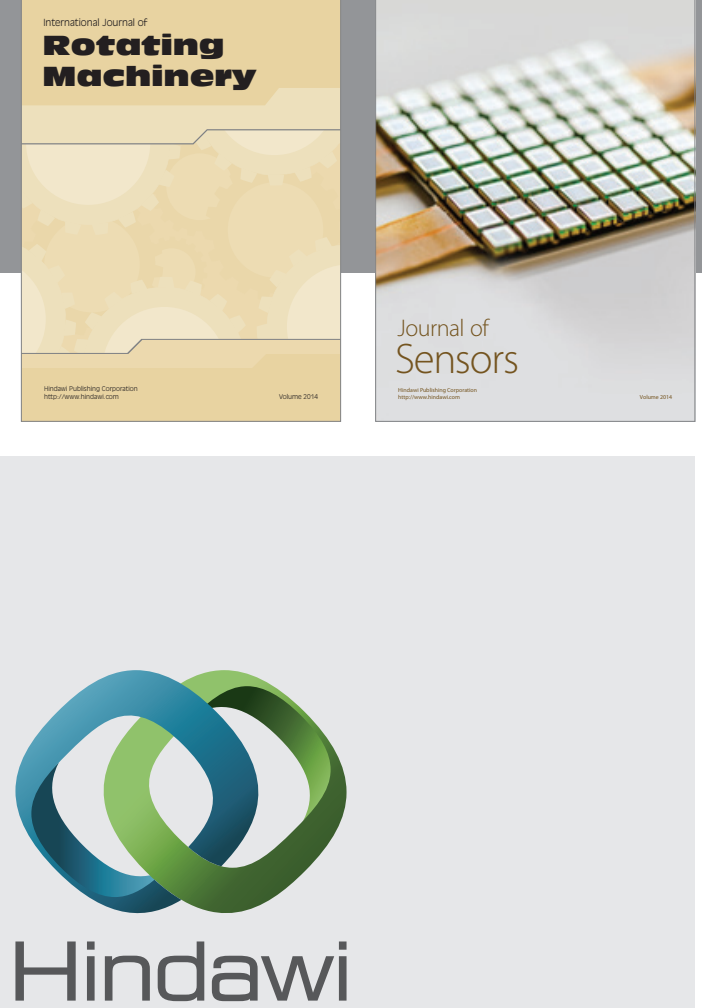

Submit your manuscripts at http://www.hindawi.com
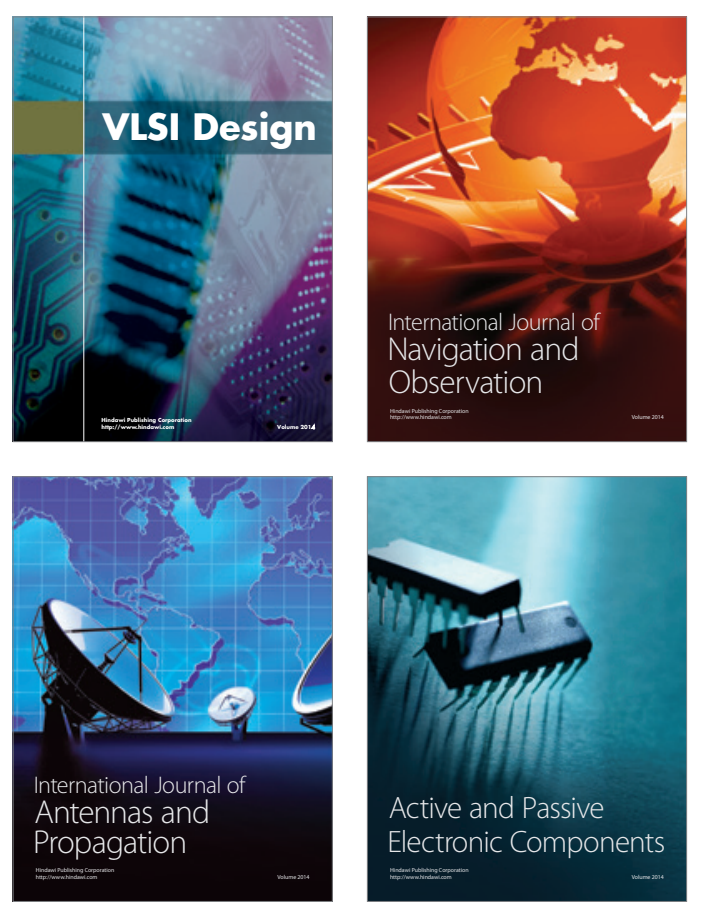
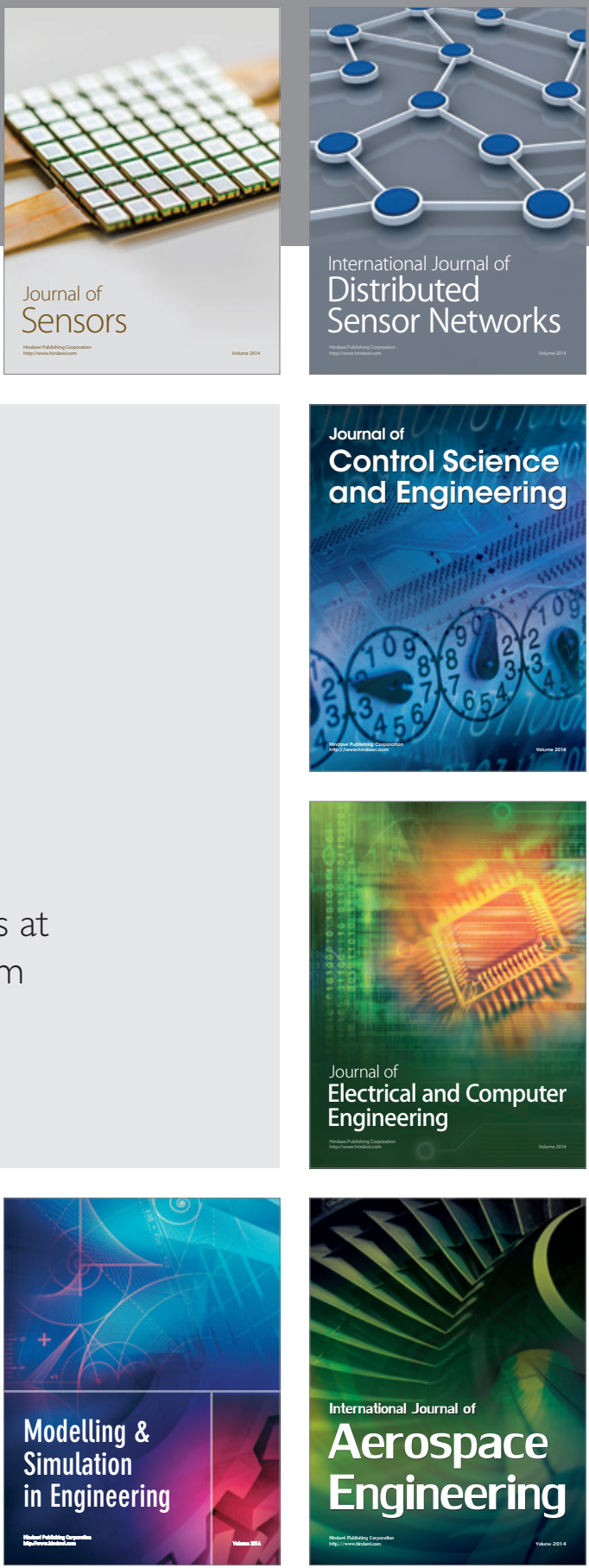

Journal of

Control Science

and Engineering
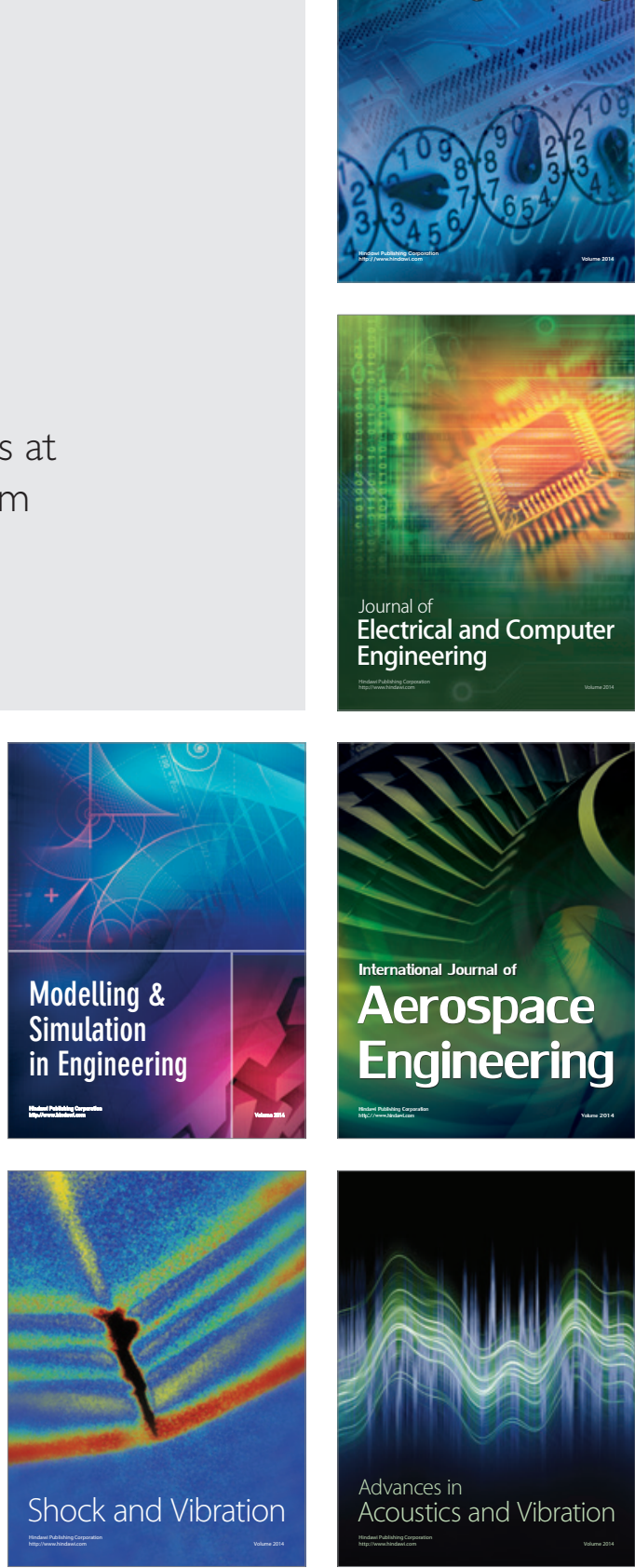